\title{
Analysis of the radiation background in CMS RPCs
}

\author{
Mircho Rodozov* On behalf of the CMS Collaboration \\ Bulgarian Academy of Sciences, \\ Institute for Nuclear Research and Nuclear Energy, 72 Tzarigradsko chaussee, Blvd. 1784 Sofia, \\ Bulgaria \\ E-mail: mrodozovecern.ch
}

\begin{abstract}
The radiation background levels in the CMS RPCs have been measured by using proton-proton collision data at $\sqrt{s}=7 \mathrm{TeV}$ collected by CMS in 2011. The dependence on the LHC instantaneous luminosity has been studied over several orders of magnitude. The background distribution in the RPC detector has been carefully analyzed. Extrapolations to the LHC design conditions are also discussed.
\end{abstract}

XI workshop on Resistive Plate Chambers and Related Detectors - RPC2012,

February 5-10, 2012

INFN Laboratori Nazionali di Frascati Italy

${ }^{*}$ Speaker. 


\section{The CMS RPCs}

The Compact Muon Solenoid (CMS) experiment [1] is one of the two general-purpose detectors observing proton-proton and heavy-ion collisions at the CERN Large Hadron Collider (LHC) [2]. The main subsystems of the CMS detector include the tracker system, the calorimetric system, and the muon system. The central feature of the Compact Muon Solenoid (CMS) apparatus is a superconducting solenoid, of $6 \mathrm{~m}$ internal diameter, providing a field of $3.8 \mathrm{~T}$. Within the field volume are a silicon pixel and strip tracker, a crystal electromagnetic calorimeter (ECAL) and a brass/scintillator hadron calorimeter (HCAL). Muons with pseudo-rapidity in the range $|\eta|$ $<2.4$ are measured with detection planes made of three technologies: Drift Tube chambers (DT), Cathode Strip Chambers (CSC) and Resistive Plate Chambers (RPC) [3].

CMS uses double-gap RPCs operating in avalanche mode [3]. Figure 1 shows a schematic view of one quarter of the CMS detector in the R-z plane (Fig.1, left) and the layout of a doublegap Barrel chamber (Fig.1, right). A double-gap is made of two single gaps which are put one on the top of the other with common read-out copper strips in the middle (Fig.1, right). The CMS RPC system is formed by central Barrel part subdivided in five wheels, and closing Endcap part, constructed from six disks. A Barrel wheel consists of four stations (RB1 to RB4), where station 1 is the innermost and station 4 - the outermost, and an Endcap disk is constructed from two stations or rings (RE2 and RE3). Each Barrel station is composed by 12 azimuthal sectors and an Endcap station by 36 azimuthal sectors. The RPC system consists of 480 Barrel chambers and 432 Endcap chambers and it covers a geometrical region up to $|\eta|<1.6$.

Background radiation levels in the CMS muon system are an important consideration in the overall performance of the system. The RPCs are employed in CMS as dedicated trigger detector. High background levels in CMS RPC could affect the trigger performance and pattern recognition of muon tracks. The 2011 proton-proton collisions data is used to study the radial, longitudinal and angular distribution of the background and the increase of the background with the LHC instantaneous luminosity. The results are in good qualitative agreement [4] with the other muon subsystems Drift Tubes (DT) and Cathode Strip Chambers (CSC).
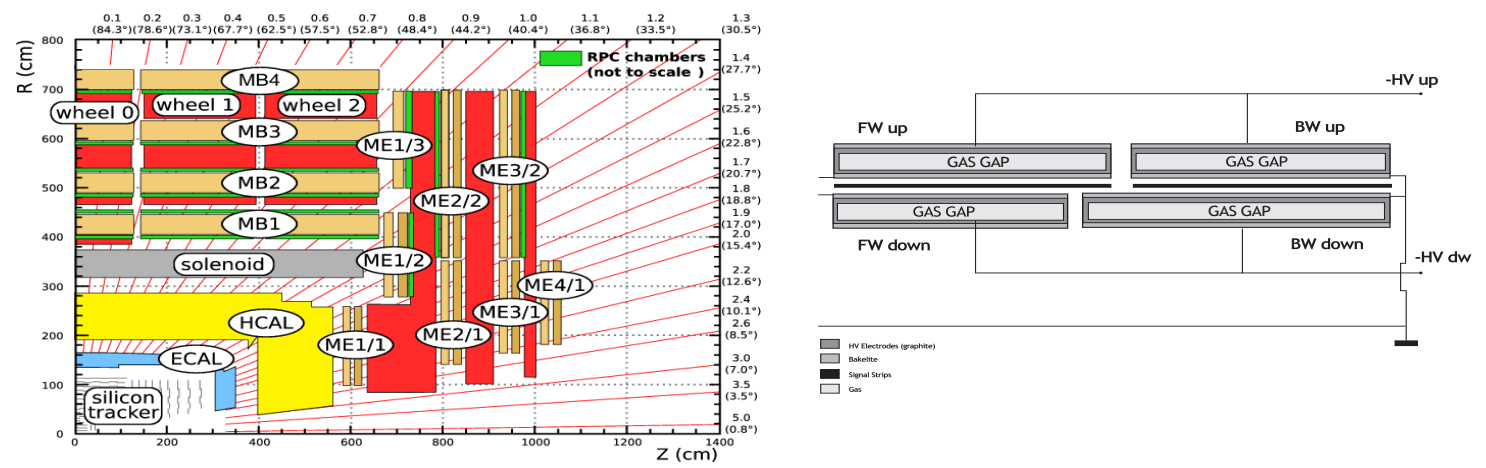

Figure 1: Left: Schematic view, in the R-z plane, of one quadrant of the CMS detector. The position of the RPC chambers is shown. Right: Schematic layout of a double-gap Barrel chamber composed by two sub-units, called rolls. The readout strip plane is also shown. 


\section{Radial distribution of the background}

The rate as a function of the instantaneous luminosity is studied for investigating the radial distribution of the background. In the Barrel the stations with highest occupancy are station 4 (RB4), mainly due to neutron return flux through the high $|\eta|$ detector regions and station 1 (RB1), mainly from particles coming from the vertex through the gaps between the Barrel and the Endcap of the calorimeters (Fig.2). In the Endcap, the innermost stations 2 are with higher occupancy as they are closer to the beam pipe with respect to the outermost stations 3 (Fig.2). Noticeable plusminus asymmetry is observed between the positive and the negative side of the Endcap (Fig.2). This asymmetry is qualitatively reproduced in the recent Fluka simulations [4] and is related to the presence of CASTOR (Centauro And STrange Object Research) forward calorimeter [5],[6] on the negative side of CMS. Measuring the background rate as function of the instantaneous luminosity is later used to extrapolate to the luminosities expected during future running of the LHC.
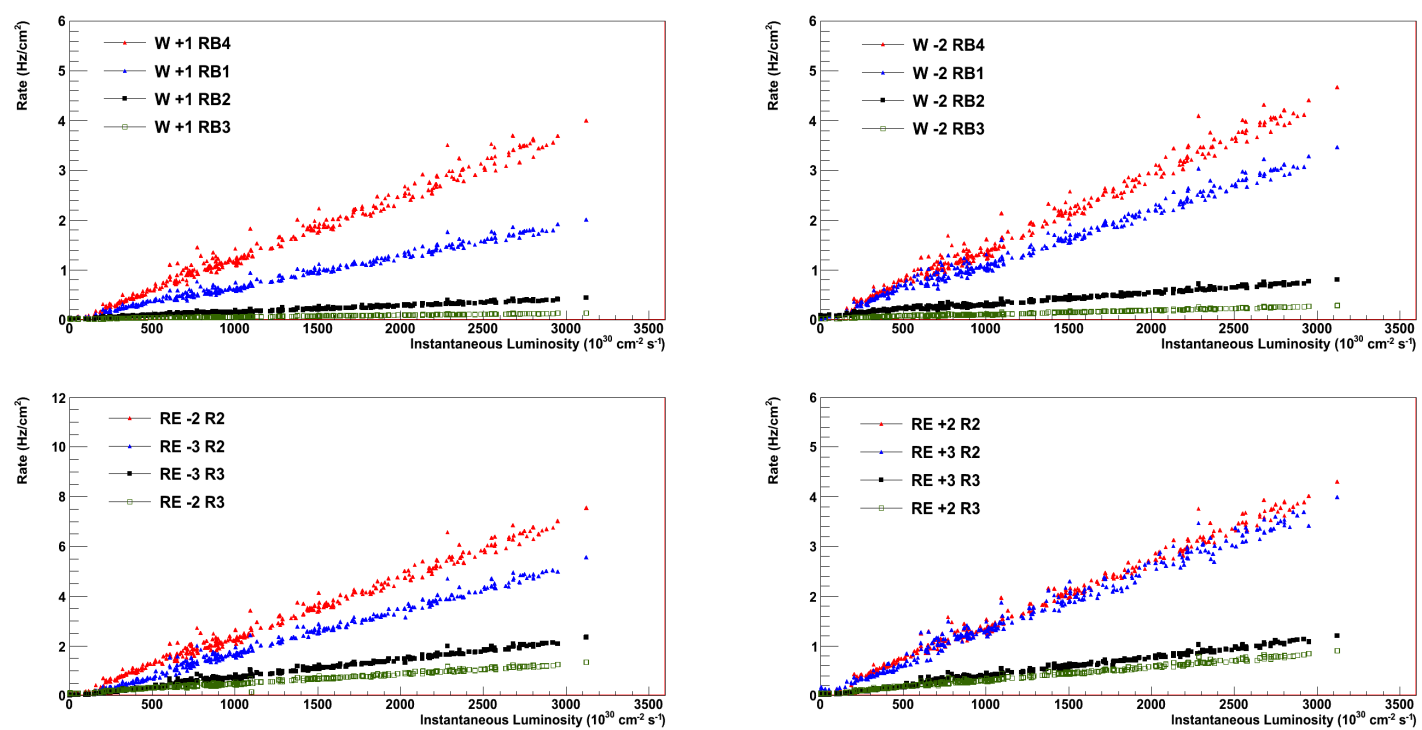

Figure 2: Background rate as a function of the instantaneous luminosity in the different stations in Barrel wheel+1 (top-left), Barrel wheel-2 (top-right), Endcap stations 2 and 3 for negative Disks 2 and 3 (bottomleft) and Endcap stations 2 and 3 for positive Disks 2 and 3 (bottom-right). Station 1 of the Endcap (Ring 1) is not installed.

\section{Angular distribution of the background}

The angular distribution of the background shows higher rate in the top sectors of station 4 of the Barrel with respect to the bottom sectors (Fig.3). For the inner Barrel stations (RB1 to RB3) the angular distribution of the background is symmetrical, as they are better shielded from outside by station 4 (RB4), while station 4 is exposed to the surrounding background. The right plot on Fig. 3 shows the top-bottom asymmetry for station 4 in the Barrel wheels, where the bottom sectors $(9,10,11)$ are better shielded by the floor and less occupied. In the Endcap (Fig.3 - left) no significant angular asymmetry is observed, as the Endcap chambers are located in between steel disks. 

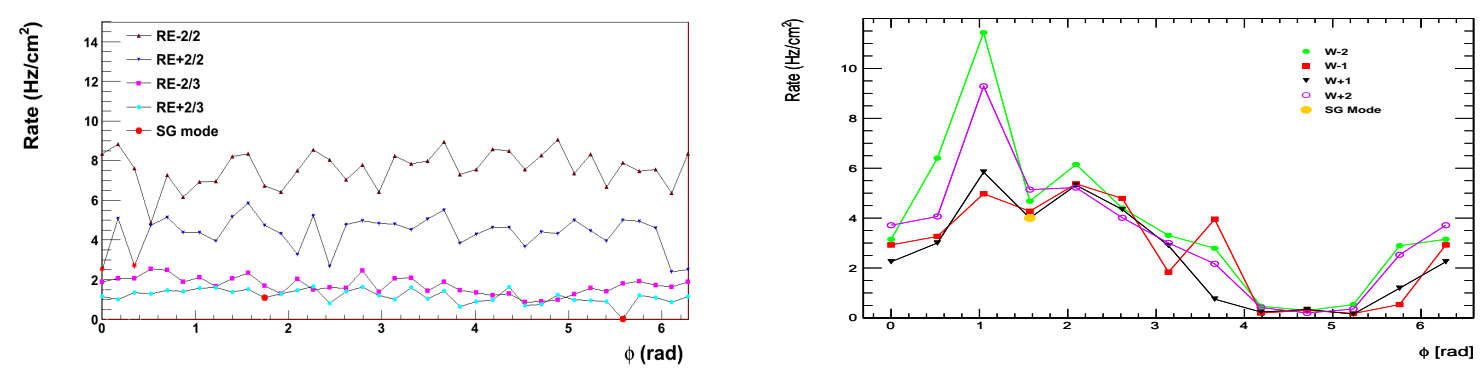

Figure 3: Left plot - Rate vs Sector for the Endcap Disks +2/-2, right plot - Rate vs Sector for four Barrel wheels station 4 only, where the higher rate in the top sectors is evident. Red entries on the left plot and orange on the right represents chambers operated in single gap mode.

\section{Longitudinal distribution of the background}

The plus-minus background asymmetry between the plus and the minus side of the Endcap has been discussed and shown on Figure 2. For the Barrel, similar studies were performed in station 4 as highly occupied, for fixed sectors (4 and 10). As only the wheel number varies, this study was aimed to reveal any longitudinal background asymmetries along the Barrel wheels, however as shown on Figure 4 the longitudinal background asymmetry in the Barrel wheels is less evident with respect to the Endcap.
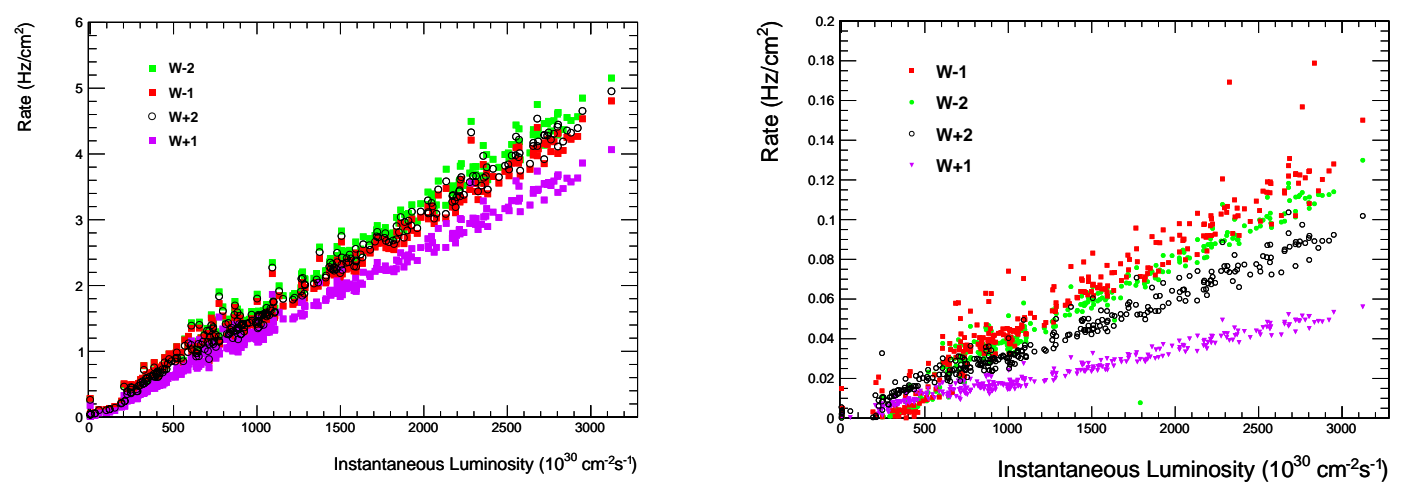

Figure 4: Rate vs Instantaneous luminosity in four Barrel wheels - sector 4 station 4 (left ), sector 10 station 4 (right).

\section{Linear extrapolation to higher luminosity}

The dependence between the background rate and luminosity is found to be linear, and linear extrapolation is used to obtain the RPC rates at luminosity $10^{34} \mathrm{~cm}^{-2} \mathrm{~s}^{-1}$. Linear extrapolation to higher luminosity gives $\sim 6 \mathrm{~Hz} / \mathrm{cm}^{2}$ as average for the system at luminosity $10^{34} \mathrm{~cm}^{-2} \mathrm{~s}^{-1}$. The highest value in the Endcap is $\sim 35-40 \mathrm{~Hz} / \mathrm{cm}^{2}$ in the $\mathrm{C}$ partitions of RE-2/2 chamber (negative Disk 2 station 2), which are with highest occupancy along the Endcap, well below the trigger limits. In addition, extrapolating the most occupied parts on Figure 2 to luminosity $10^{34} \mathrm{~cm}^{-2} \mathrm{~s}^{-1}$ (the red curves) gives $\sim 12 \mathrm{~Hz} / \mathrm{cm}^{2}$ for Wheel+1 station 4 (top-left), $\sim 14 \mathrm{~Hz} / \mathrm{cm}^{2}$ for Wheel-2 station 4 
(top-right), $\sim 23 \mathrm{~Hz} / \mathrm{cm}^{2}$ for Endcap Disk-2 station 2 (bottom-left) and $\sim 13 \mathrm{~Hz} / \mathrm{cm}^{2}$ for Endcap Disk+2 station 2 .

\section{References}

[1] CMS Collaboration, The CMS experiment at the CERN LHC, J. Instrum. 3 (2008) S08004.

[2] L. Evans and P. Bryant, LHC Machine J. Instrum., vol. 3, p. S08001, 2008, doi:10.1088/1748-0221/3/08/S08001

[3] CMS Collaboration, The Muon Project Technical Design Report, CERN/LHCC 97-32 (1997)

[4] CMS Collaboration, The performance of the CMS muon detector using pp collisions at $\sqrt{s}=7 \mathrm{TeV}$ at LHC , CMS MUO-11-001, paper to be published.

[5] CMS Collaboration, Detector Performance and Software, Technical Design Report Vol. I, CERN/LHCC 2006-01, February 2006.

[6] http://cmsdoc.cern.ch/castor/

[7] S. Costantini, Calibration of the RPC working voltage in the CMS experiment, these proceedings

[8] R. Hadjiiska, The CMS RPC performance and simulation, these proceedings 